\title{
DYNAMO: An upclose view of turbulent, clumpy galaxies
}

\section{Deanne Fisher}

Swinburne University of Technology, Australia

\begin{abstract}
Over 2/3 of all star formation in the Universe occurs in gas-rich, super-high pressure clumpy galaxies in the epoch of redshift $z \sim 1-3$. However, because these galaxies are so distant we are limited in the information available to study the properties of star formation and gas in these systems. I will present results using a sample of extremely rare, nearby galaxies (called DYNAMO) that are very well matched in gas fraction $\left(f_{\text {gas }} \sim 20-80 \%\right)$, kinematics (rotating disks with velocity dispersions ranging $20-100 \mathrm{~km} / \mathrm{s}$ ), structure (exponential disks) and morphology (clumpy star formation) to high-z main-sequence galaxies. We therefore use DYNAMO galaxies as laboratories to study the processes inside galaxies in the dominate mode of star formation in the Universe. In this talk I will report on results from our programs with HST, ALMA, Keck, and NOEMA for DYNAMO galaxies that are aimed at testing models of star formation. We have discovered of an inverse relationship between gas velocity dispersion and molecular gas depletion time. This correlation is directly predicted by theories of feedback-regulated star formation; conversely, predictions of models in which turbulence is driven by gravity only are not consistent with our data. I will also show that feedback-regulated star formation can explain the redshift evolution of galaxy star formation efficiency. I will also present results from a recently acquired map of $\mathrm{CO}(2-1)$ in a clumpy galaxy with resolution less than $200 \mathrm{pc}$. With maps such as these we can begin to study these super giant star forming clumps at scales that are more comparable to local surveys. I will show results for the star formation efficiency of clumps, the boundedness of clumps of molecular gas, and discuss links between star formation efficiency and formation of clumps of stellar mass. The details of clumpy systems are a direct constraint of the results of simulations, especially on the nature of feedback in the high density environments of star formation that dominate the early Universe.
\end{abstract}

